\title{
Antimicrobial activities of isolated probiotics and their metabolites against some pathogenic microorganisms
}

\author{
Mohammed Abu Sayeed \\ Dept. of Pharmacy, International Islamic University Chittagong, Bangladesh \\ Altaf Hossen, Repon Saha \\ Dept. of Pharmacy, East West University, Dhaka, Bangladesh \\ and Md. Jakaria \\ Dept. of Pharmacy, Southern University Bangladesh, Bangladesh
}

\begin{abstract}
The study was aimed to finding the antimicrobial activities among probiotics isolated from different yoghurts and their metabolites against some common bacterial pathogens. The nutrient agar media overlay method (Disc diffusion Method) was used to determine the presence of antibacterial effects among the isolated probiotics. Probiotics produced potential antibacterial activities against several pathogenic bacteria and fungi. The maximum antibacterial property (13.5 $\mathrm{mm}$ of zone of inhibition) of bacterial strain found against Salmonella paratyphi. Conversly, bacterial metabolites produced maximum effect (10.3 mm of zone of inhibition) against Staphylocuccos aureaus. The antibacterial effect is one of the most important criteria for probiotics selection, and the verified antibacterial activities of the probiotics supports the development of these functional foods as a key to the enhancement of health in the consuming public.
\end{abstract}

Keywords Probiotics, yoghurts, Salmonella paratyphi, Staphylocuccos aureaus, antimicrobial effects

Paper type Research paper

\section{Introduction}

Probiotics defined as the microorganisms (in most cases, bacteria) that are supposed to provide health benefits when consumed (Hill et al., 2014; Rijkers, 2011). They are also known as "friendly bacteria" or "good bacteria." Nowadays, the term probiotic is used to name ingested microorganisms associated with benefits for humans and animals (Magdalena, 2006). In medicine, probiotics are living microorganisms, which are incorporated into

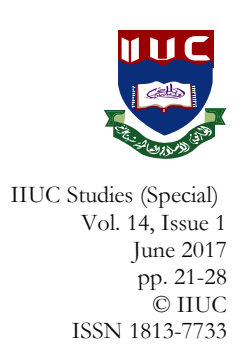


different kinds of foods due to their health-promoting effects. Due to lack of no side effects, probiotics are used for long times in food ingredients for human as well as to feed the animals. In addition, probiotics are suitable because of being naturally found in the intestinal tract as intestinal flora of healthy humans and in foods.

In the present day, the members of the genera Lactobacillus, Bifidobacterium and Streptococcus are the most familiar probiotics used in commercial fermented and non-fermented dairy products (Heller, 2001). The properties against bacteria are one of the most significant selection criteria for probiotics (Klaenhammer, \& Kullen, 1999). The antimicrobial effects of lactic acid bacteria are formed by producing some substances such as organic acids (lactic, acetic, propionic acids), carbon dioxide, hydrogen peroxide, diacetyl, low molecular weight antimicrobial substances and bacteriocins (Quwehand \& Vesterlund, 2006).

A number of studies have found probiotic consumption to be useful in the treatment of many types of diarrhea, including antibiotic-associated diarrhea in adults, travelers' diarrhea, and diarrheal diseases in young children caused by rotaviruses. The most commonly studied probiotic species in these studies have been found to be Lactobacillus GG, L. casei, B. bifidum and $S$. thermophilus (Isolauri et al., 1991). The aim of this study was to determine the presence of antibacterial effects among the probiotics isolated from different yoghurts against some common bacterial pathogens.

\section{Materials and Methods}

\section{a. Yoghurts, Probiotics, Media and Pathogen Strains}

Yogurt was collected from different brand sources such as; Arong sour yoghurt, Rosmela sour and sweet yoghurt, Rajdani sweet yoghurt and Eglo sweet yoghurt. $100 \mu$ l of each samples were taken by the micropipette from each tube, then incorporate into Petri-dish. Samples were spread out into Petri-dishes. Then nutrient agar media was to be given in each Petridish and kept in incubator for 24-48 hour. Cultures in solid media were visually inspected for growth rate and colony characteristics. Several types of colonies were seen in the Petri-dishes. The Probiotic properties were determined by several tests like Resistance to low $\mathrm{pH}$ and Resistance to $1 \%$ bile salt etc. Carbohydrate Fermentation Test, Glucose fermentation test, Tolerance Against 6.5\% Sodium Chloride and protein nature tests were also performed. The isolated bacteria were gram stained for the study of microscopic morphology. The test pathogens were gram positive bacteria like Bacillus sereus, Bacillus subtilis, Gram negative bacteria Salmonella 
paratyphi, Vibrio parahemolyticus, Staphylocuccos aureaus, Escherichia coli, Shigella dysenteriae, Sarcina lutea and fungi like Candida albicans, Asergillus niger etc.

\section{b. Determination of Antimicrobial Effects}

The selected pathogens were maintained in nutrient agar media at $25^{\circ} \mathrm{C}$. The test antimicrobial activity, prepared standard discs (isolates) as the concentration of $20 \mu \mathrm{l}$ of each sample.

Determination of antimicrobial activity of isolated metabolites against different pathogenic microorganisms from subculture also was performed. The blank discs were kept in covered Petri dishes and then subjected to dry heat sterilization for $1 \mathrm{hr}$ at $150^{\circ} \mathrm{C}$. After completion of sterilization, both the sterilized glass materials and discs were kept in a laminar hood for 30 minutes. UV light was switched on before working in laminar hood to avoid any accidental contamination. Isolated metabolites were resuspended by adding $0.5 \mathrm{ml}$ phosphate buffer $\left(\mathrm{p}^{\mathrm{H}_{-}}\right.$-7.1) and shaking vigorously for dissolve of metabolites. For each disc $20 \mu \mathrm{l}$ sample were

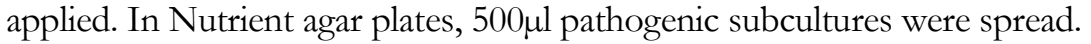

The prepared metabolites discs were placed gently on the solidifier agar plates where contains the pathogenic organism with the help of a sterile forcep to ensure complete contact with medium surface. This was sufficient time for the material to diffuse to a considerable area of the medium. Finally, the plates were incubated at $37^{\circ} \mathrm{c}$ for $24 \mathrm{hrs}$ (Barefoot \& Klaenhammer, 1984; Wolfson, 1999).

\section{Results and Discussion}

\section{a. Macroscopic and Microscopic Properties of Isolated Probiotics}

The probiotics isolates were cream colored, circular, convex and moist with smooth edges. Microscopic smear of gram staining of the Colonies showed gram-positive, non-sporeforming short bacilli in pairs or in chains. The results were those expected of Steptococci sp, Lactobacillus sp and Bifidobacterium sp. found in yoghurts. When slides were observed under light microscope, it was shown 8 (eight) colonies gave blue (violet) colour among 15 colonies. So, they were gram positive (+ve) bacteria and other colonies were discarded. Selected colonies were given new code (Table 1).

Table 1. Gram staining result of isolates

\begin{tabular}{llll}
\hline Strain code & Colour & Gram stain & New code \\
\hline ATY-1 & Blue & + & 1 \\
ATY-2 & Blue & + & 2 \\
ATY-3 & Red & - & $\times$ \\
ATY-4 & Blue & + & 3 \\
\hline
\end{tabular}


24 IIUC STUDIES, 14 (Issue 1, Special)

\begin{tabular}{llll}
\hline Strain code & Colour & Gram stain & New code \\
\hline & & & \\
RTY-1 & Blue & + & 4 \\
RTY-2 & Red & - & $\times$ \\
RTY-3 & Red & - & $\times$ \\
RTY-4 & Blue & + & 5 \\
EMY-1 & Blue & - & 6 \\
EMY-2 & Red & - & $\times$ \\
EMY-3 & Red & + & $\times$ \\
RMY-1 & Blue & + & 7 \\
RMY-2 & Blue & - & 8 \\
RMY-3 & Red & - & $\times$ \\
DMY & Red & & $\times$ \\
\hline
\end{tabular}

\section{b. Antimicrobial Activities of Selected Strains}

All strains of probiotic bacteria were found from experiments. These strains were catalase negative, survived against low $\mathrm{pH}(\mathrm{pH} 3.0 \& 1.0)$ during 3 hours and against $1 \%$ bile salt during 4 hours, show antimicrobial activity against different types of pathogenic bacteria. The bacterial strains had antagonistic effect against pathogenic bacteria. The zone of inhibition in $\mathrm{mm}$ was shown in Table 2 . In antimicrobial activity test, all 8 strains were given effect against all pathogenic bacteria. But strain no. 1 did not given effect against Bacillus subtilis. Beside Bacillus sereus, bacterial strain no. 6 \& 1 produced maximum zone of inhibition $(10.75 \mathrm{~mm} \& 9.6 \mathrm{~mm})$ but against Bacillus subtils, no. 3 \& 2 strains produced maximum zone of inhibition $(8.35 \mathrm{~mm} \& 7.45 \mathrm{~mm})$. Maximum inhibition of Salmonella paratyphi occurred by $7 \& 6$ number of strains and inhibition area were $13.55 \mathrm{~mm} \& 9.35 \mathrm{~mm}$ respectively. Strains no. $7 \& 8$ produced maximum inhibition $(12.85 \mathrm{~mm} \&$ $10.55 \mathrm{~mm})$ against Vibrio parabemolyticus. For Staphylococcus aureaus, strains 5 \& 6 produced higher inhibition $(15.00 \mathrm{~mm} \& 10.9 \mathrm{~mm})$ than other strains. Against E.coli, strains $3 \& 5$ produced maximum inhibition $(12.85 .55 \mathrm{~mm} \&$ $12.15 \mathrm{~mm}$ ) respectively. Higher inhibitions of Shigella dysenteriae occurred by 4 \& 7 number strains and inhibited area are $8.3 \mathrm{~mm}$ and $8.05 \mathrm{~mm}$. Strains $8 \&$ 7 produced maximum inhibition $(9.2 \mathrm{~mm} \& 9.1 .6 \mathrm{~mm})$ against Sarcina lutea. For Candida albicans, higher inhibition are caused by strains $3 \& 4$ respectively and inhibited area in slide calipers were $10.95 \mathrm{~mm} \& 8.55 \mathrm{~mm}$. Against Apergillus niger, strains $5 \& 4$ produecd maximum zone of inhibition $(7.8 \mathrm{~mm} \& 7.75 \mathrm{~mm})$ than others strains. Among all strains, $15.0 \mathrm{~mm}$ was maximum zone of inhibition against Staphylococcus aureaus and effective strains are 8 number strain.

\section{c. Antimicrobial Activities of Bacterial Metabolites}

Bacteriocin or bactarial metabolites show significant inhibitory action against pathogenic bacteria. Isolated metabolites were diluted by using 
$500 \mu \mathrm{l}$ phosphate buffer ( $\mathrm{pH}-7.1)$. Disc contains $20 \mu \mathrm{l}$ metabolites samples and $500 \mu \mathrm{l}$ pathogenic bacteria were spread in agar plate. Then all dishes were kept in incubator for 24 hour's. Isolated bacterial metabolites were considered for antimicrobial test against 10 pathogenic bacteria. We observed that all 8 different metabolites were produced zone of inhibition against given pathogenic bacteria but 3, 4 number metabolites have no any activity against Shigella dysenteriae. Antibacterial activity of metabolites is given in Table 3.

Against Bacillus sereus, metabolites $6 \& 2$ produced maximum zone of inhibition $(8.05 \mathrm{~mm} \& 8.2 \mathrm{~mm})$. Against Bacillus subtilis, $2 \& 3$ number metabolites produced maximum zone of inhibition $(9.8 \mathrm{~mm} \& 9.8 \mathrm{~mm})$. Maximum inhibition of Salmonella paratyphi occurred by $4 \& 6$ number of metabolites and inhibition area were $8.8 \mathrm{~mm} \& 8.4 \mathrm{~mm}$ respectively. Metabolites $7 \& 5$ produced maximum inhibition $(8.05 \mathrm{~mm} \& 7.6 \mathrm{~mm})$ against Vibrio parahemolyticus. For Staphylococcus aureaus, metabolites 2 \& 3 produced higher inhibition $(9.05 \mathrm{~mm} \& 8.9 \mathrm{~mm})$ than other metabolites. Against E. coli, matabolites $8 \& 7$ produced maximum inhibition $(9.55 \mathrm{~mm}$ $\& 7.75 \mathrm{~mm}$ ) respectively. Higher inhibition of Shigella dysenteriae occurred by $8 \& 1$ number metabolites and inhibition area were $8.55 \mathrm{~mm}$ and 7.6 $\mathrm{mm}$. Metabolites $5 \& 1$ produced maximum inhibition $(8.1 \mathrm{~mm} \& 7.6 \mathrm{~mm})$ against Sarcina lutea. For Candida albicans, higher inhibition were caused by metabolites $8 \& 7$ respectively and inhibition area in slide calipers were $9.1 \mathrm{~mm} \& 8.3 \mathrm{~mm}$. Against Apergillus niger, metabolites $8 \& 7$ produced maximum zone of inhibition $(7.7 \mathrm{~mm} \& 7.35 \mathrm{~mm})$ than others metabolites. Among all metabolites, $9.8 \mathrm{~mm}$ is maximum zone of inhibition against Bacillus subtilis and effective metabolites were $2 \& 3$ numbers metabolite.

Table 2: Zone of inhibition of isolates against pathogenic bacteria

\begin{tabular}{|c|c|c|c|c|c|c|c|c|c|}
\hline \multirow{2}{*}{\multicolumn{2}{|c|}{$\begin{array}{l}\text { Pathgenic } \\
\text { Organisms }\end{array}$}} & \multicolumn{8}{|c|}{ Zone of Inhibition in $\mathrm{mm}$} \\
\hline & & & & Strair & Code of & elected & acteria & & \\
\hline Name & Code & 1 & 2 & 3 & 4 & 5 & 6 & 7 & 8 \\
\hline $\begin{array}{l}\text { Bacillus } \\
\text { sereus }\end{array}$ & 1 & $\begin{array}{c}9.6 \\
\pm 4 \\
8\end{array}$ & $\begin{array}{c}6.8 \pm 0 \\
.6\end{array}$ & $\begin{array}{c}6.9 \pm 0 \\
8\end{array}$ & $\begin{array}{c}7.3 \pm 0 \\
.0\end{array}$ & $\begin{array}{c}7.4 \pm 1 \\
.0\end{array}$ & $\begin{array}{c}10.7 \pm \\
5.0\end{array}$ & $\begin{array}{c}6.65 \pm \\
1.1\end{array}$ & $\begin{array}{c}9.6 \pm 4 \\
.4\end{array}$ \\
\hline $\begin{array}{l}\text { Bacillus } \\
\text { subtilis }\end{array}$ & 3 & - & $\begin{array}{c}7.4 \pm 2 \\
.7\end{array}$ & $\begin{array}{c}8.35 \pm \\
3.3\end{array}$ & $\begin{array}{c}7.4 \pm 0 \\
.2\end{array}$ & $\begin{array}{c}7.0 \pm 0 \\
.6\end{array}$ & $\begin{array}{c}6.2 \pm 0 \\
.2\end{array}$ & $\begin{array}{c}6.65 \pm \\
0.3\end{array}$ & $\begin{array}{c}6.1 \pm 0 \\
.0\end{array}$ \\
\hline $\begin{array}{l}\text { Salmonell } \\
\text { a } \\
\text { paratyphi }\end{array}$ & 4 & $\begin{array}{c}7.9 \\
\pm 0 \\
8\end{array}$ & $\begin{array}{c}7.5 \pm 1 \\
.6\end{array}$ & $\begin{array}{c}6.33 \pm \\
0.5\end{array}$ & $\begin{array}{c}6.7 \pm 1 \\
.2\end{array}$ & $\begin{array}{c}7.4 \pm 1 \\
.4\end{array}$ & $\begin{array}{c}9.35 \pm \\
3.3\end{array}$ & $\begin{array}{c}13.5 \pm \\
8.9\end{array}$ & $\begin{array}{c}11.5 \pm \\
0.0\end{array}$ \\
\hline $\begin{array}{c}\text { Vibrio } \\
\text { parabemol } \\
\text { yticus }\end{array}$ & 6 & $\begin{array}{c}7.95 \\
\pm 2 . \\
3\end{array}$ & $\begin{array}{c}6.3 \pm 0 \\
.0\end{array}$ & $\begin{array}{c}6.4 \pm 0 \\
0\end{array}$ & $\begin{array}{c}6.3 \pm 0 \\
.1\end{array}$ & $\begin{array}{c}6.65 \pm \\
1.1\end{array}$ & $\begin{array}{c}7.85 \pm \\
2.5\end{array}$ & $\begin{array}{c}12.8 \pm \\
4.2\end{array}$ & $\begin{array}{c}10.5 \pm \\
4.6\end{array}$ \\
\hline $\begin{array}{c}\text { Stapbyloc } \\
\text { uccos } \\
\text { aureaus }\end{array}$ & 8 & $\begin{array}{c}7.4 \\
\pm 2 \\
6\end{array}$ & $\begin{array}{c}8.65 \pm \\
2.3\end{array}$ & $\begin{array}{c}7.35 \pm \\
0.5\end{array}$ & $\begin{array}{c}9.15 \pm \\
1.7\end{array}$ & $\begin{array}{c}15 \pm 0 \\
6\end{array}$ & $\begin{array}{c}10.9 \pm \\
0.9\end{array}$ & $\begin{array}{c}6.1 \pm 0 \\
.0\end{array}$ & $\begin{array}{c}7.5 \pm 0 \\
.8\end{array}$ \\
\hline
\end{tabular}




\section{IIUC STUDIES, 14 (Issue 1, Special)}

\begin{tabular}{|c|c|c|c|c|c|c|c|c|c|}
\hline \multirow{2}{*}{\multicolumn{2}{|c|}{$\begin{array}{l}\text { Pathgenic } \\
\text { Organisms }\end{array}$}} & \multicolumn{8}{|c|}{ Zone of Inhibition in $\mathrm{mm}$} \\
\hline & & \multicolumn{8}{|c|}{ Strain Code of Selected Bacteria } \\
\hline Name & Code & 1 & 2 & 3 & 4 & 5 & 6 & 7 & 8 \\
\hline E.coli & 9 & $\begin{array}{c}7.65 \\
\pm 1 \\
1\end{array}$ & $\begin{array}{c}7.9 \pm 0 \\
.4\end{array}$ & $\begin{array}{l}12.85 \\
\pm 4.2\end{array}$ & $\begin{array}{c}9.8 \pm 3 \\
.7\end{array}$ & $\begin{array}{c}12.1 \pm \\
0.5\end{array}$ & $\begin{array}{c}7.7 \pm 0 \\
.4\end{array}$ & $\begin{array}{c}7.6 \pm 0 \\
.0\end{array}$ & $\begin{array}{c}6.8 \pm 0 \\
.0\end{array}$ \\
\hline $\begin{array}{c}\text { Shigella } \\
\text { dysenteriae }\end{array}$ & 10 & $\begin{array}{c}7.55 \\
\pm 0 \\
5\end{array}$ & $\begin{array}{c}7.5 \pm 2 \\
.2\end{array}$ & $\begin{array}{c}7.1 \pm 0 \\
0\end{array}$ & $\begin{array}{c}8.3 \pm 2 \\
.8\end{array}$ & $\begin{array}{c}7.05 \pm \\
1.7\end{array}$ & $\begin{array}{c}6.4 \pm 0 \\
.0\end{array}$ & $\begin{array}{c}8.05 \pm \\
0.7\end{array}$ & $\begin{array}{c}6.5 \pm 0 \\
.8\end{array}$ \\
\hline $\begin{array}{c}\text { Sarcina } \\
\text { lutea }\end{array}$ & 12 & $\begin{array}{c}7.8 \\
\pm 0 \\
0\end{array}$ & $\begin{array}{c}6.25 \pm \\
0.3\end{array}$ & $\begin{array}{c}6.3 \pm 0 \\
0\end{array}$ & $\begin{array}{c}7.6 \pm 0 \\
.0\end{array}$ & $\begin{array}{c}6.15 \pm \\
0.1\end{array}$ & $\begin{array}{c}7.8 \pm 0 \\
.0\end{array}$ & $\begin{array}{c}9.1 \pm 0 \\
.0\end{array}$ & $\begin{array}{c}9.2 \pm 0 \\
.0\end{array}$ \\
\hline $\begin{array}{l}\text { Candida } \\
\text { albicans }\end{array}$ & 15 & $\begin{array}{c}8.5 \\
\pm 1 \\
2\end{array}$ & $\begin{array}{c}9.5 \pm 2 \\
.4\end{array}$ & $\begin{array}{l}10.95 \\
\pm 0.3\end{array}$ & $\begin{array}{c}8.85 \pm \\
0.5\end{array}$ & $\begin{array}{c}7.7 \pm 1 \\
.0\end{array}$ & $\begin{array}{l}8.2 \pm 0 \\
.2\end{array}$ & $\begin{array}{c}6.15 \pm \\
0.1\end{array}$ & $\begin{array}{c}7.35 \pm \\
2.4\end{array}$ \\
\hline $\begin{array}{c}\text { Asergillus } \\
\text { niger }\end{array}$ & 16 & $\begin{array}{c}6.75 \\
\pm 0 \\
3\end{array}$ & $\begin{array}{c}7.2 \pm 0 \\
.0\end{array}$ & $\begin{array}{c}7.2 \pm 1 \\
8\end{array}$ & $\begin{array}{c}7.75 \pm \\
1.1\end{array}$ & $\begin{array}{c}7.8 \pm 1 \\
.4\end{array}$ & $\begin{array}{c}7.7 \pm 0 \\
.0\end{array}$ & $\begin{array}{c}7.7 \pm 0 \\
.8\end{array}$ & $\begin{array}{c}6.8 \pm 0 \\
.8\end{array}$ \\
\hline
\end{tabular}

Values are presented as the Mean $\pm S E(n=3)$

Table 3: Inhibitory result of metabolites against pathogenic bacteria

\begin{tabular}{|c|c|c|c|c|c|c|c|c|c|}
\hline \multirow{2}{*}{\multicolumn{2}{|c|}{$\begin{array}{l}\text { Pathgenic } \\
\text { Organisms }\end{array}$}} & \multicolumn{8}{|c|}{ Zone of Inhibition in $\mathrm{mm}$} \\
\hline & & \multicolumn{8}{|c|}{ Strain Code of Isolated Metabolites } \\
\hline Name & Code & 1 & 2 & 3 & 4 & 5 & 6 & 7 & 8 \\
\hline $\begin{array}{c}\text { Bacillus } \\
\text { sereus }\end{array}$ & 1 & $\begin{array}{c}6.85 \\
\pm 0 \\
1\end{array}$ & $\begin{array}{c}8.2 \\
\pm 0 \\
8\end{array}$ & $\begin{array}{c}6.75 \pm \\
1.1\end{array}$ & $\begin{array}{c}7.9 \pm 0 \\
.0\end{array}$ & $\begin{array}{c}6.95 \pm \\
1.3\end{array}$ & $\begin{array}{c}8.05 \pm \\
0.3\end{array}$ & $\begin{array}{c}7.6 \pm 1 \\
.0\end{array}$ & $\begin{array}{c}6.7 \pm \\
1.2\end{array}$ \\
\hline $\begin{array}{l}\text { Bacillus } \\
\text { subtilis }\end{array}$ & 3 & $\begin{array}{c}7.8 \\
\pm 0 \\
0\end{array}$ & $\begin{array}{c}9.8 \\
\pm 1 \\
5\end{array}$ & $\begin{array}{c}9.8 \pm 1 \\
.5\end{array}$ & $\begin{array}{c}7.25 \pm \\
0.7\end{array}$ & $\begin{array}{c}7.8 \pm 1 \\
.8\end{array}$ & $\begin{array}{c}7.5 \pm 0 \\
.8\end{array}$ & $\begin{array}{c}7.35 \pm \\
1.9\end{array}$ & $\begin{array}{c}7.9 \pm \\
1.4\end{array}$ \\
\hline $\begin{array}{l}\text { Salmonella } \\
\text { paratyphi }\end{array}$ & 4 & $\begin{array}{c}7.25 \\
\pm 0 \\
9\end{array}$ & $\begin{array}{l}7.2 \\
5 \pm \\
0.9\end{array}$ & $\begin{array}{c}8.05 \pm \\
1.1\end{array}$ & $\begin{array}{c}8.8 \pm 0 \\
.8\end{array}$ & $\begin{array}{c}8.65 \pm \\
3.8\end{array}$ & $\begin{array}{c}7.6 \pm 1 \\
.4\end{array}$ & $\begin{array}{c}8.4 \pm 1 \\
.4\end{array}$ & $\begin{array}{c}8.4 \pm \\
1.6\end{array}$ \\
\hline $\begin{array}{c}\text { Vibrio } \\
\text { parahemol } \\
\text { yticus }\end{array}$ & 6 & $\begin{array}{c}7.4 \\
\pm 2 \\
6\end{array}$ & $\begin{array}{c}7.1 \\
\pm 2 \\
0\end{array}$ & $\begin{array}{c}6.95 \pm \\
0.3\end{array}$ & $\begin{array}{c}7.15 \pm \\
1.9\end{array}$ & $\begin{array}{c}7.6 \pm 2 \\
.6\end{array}$ & $\begin{array}{c}7.35 \pm \\
0.1\end{array}$ & $\begin{array}{c}8.05 \pm \\
0.5\end{array}$ & $\begin{array}{c}7.5 \pm \\
2.5\end{array}$ \\
\hline $\begin{array}{c}\text { Staphylocu } \\
\text { ccos } \\
\text { aureaus }\end{array}$ & 8 & $\begin{array}{c}9.05 \\
\pm 3 \\
0\end{array}$ & $\begin{array}{l}10 . \\
3 \pm \\
0.0\end{array}$ & $\begin{array}{c}8.9 \pm 0 \\
.0\end{array}$ & $\begin{array}{c}6.85 \pm \\
1.1\end{array}$ & $\begin{array}{c}6.65 \pm \\
1.1\end{array}$ & $\begin{array}{c}7.35 \pm \\
1.9\end{array}$ & $\begin{array}{c}8.1 \pm 0 \\
.4\end{array}$ & $\begin{array}{c}7.0 \pm \\
3.3\end{array}$ \\
\hline E.coli & 9 & $\begin{array}{c}7.6 \\
\pm 2 . \\
2\end{array}$ & $\begin{array}{l}7.5 \\
5 \pm \\
2.7\end{array}$ & $\begin{array}{c}6.5 \pm 0 \\
.8\end{array}$ & $\begin{array}{c}6.45 \pm \\
0.5\end{array}$ & $\begin{array}{c}6.15 \pm \\
0.1\end{array}$ & $\begin{array}{c}6.7 \pm 1 \\
.2\end{array}$ & $\begin{array}{c}7.7 \pm 0 \\
.9\end{array}$ & $\begin{array}{c}9.5 \pm \\
2.0\end{array}$ \\
\hline $\begin{array}{c}\text { Shigella } \\
\text { dysenteriae }\end{array}$ & 10 & $\begin{array}{c}7.6 \\
\pm 1 \\
4\end{array}$ & $\begin{array}{c}6.9 \\
\pm 0 \\
0\end{array}$ & - & - & $\begin{array}{c}6.2 \pm 0 \\
.2\end{array}$ & $\begin{array}{c}6.45 \pm \\
0.5\end{array}$ & $\begin{array}{c}6.8 \pm 0 \\
.0\end{array}$ & $\begin{array}{c}8.5 \pm \\
0.7\end{array}$ \\
\hline
\end{tabular}




\begin{tabular}{|c|c|c|c|c|c|c|c|c|c|}
\hline \multirow{2}{*}{\multicolumn{2}{|c|}{$\begin{array}{l}\text { Pathgenic } \\
\text { Organisms }\end{array}$}} & \multicolumn{8}{|c|}{ Zone of Inhibition in $\mathrm{mm}$} \\
\hline & & \multicolumn{8}{|c|}{ Strain Code of Isolated Metabolites } \\
\hline Name & Code & 1 & 2 & 3 & 4 & 5 & 6 & 7 & 8 \\
\hline $\begin{array}{c}\text { Sarcina } \\
\text { lutea }\end{array}$ & 12 & $\begin{array}{c}7.6 \\
\pm 1 . \\
8\end{array}$ & $\begin{array}{c}6.7 \\
\pm 0 . \\
2\end{array}$ & $\begin{array}{c}7.3 \pm 1 \\
.2\end{array}$ & $\begin{array}{c}7.35 \pm \\
0.9\end{array}$ & $\begin{array}{c}8.1 \pm 3 \\
.6\end{array}$ & $\begin{array}{c}7.3 \pm 1 \\
.6\end{array}$ & $\begin{array}{c}6.95 \pm \\
0.5\end{array}$ & $\begin{array}{c}7.0 \pm \\
1.6\end{array}$ \\
\hline $\begin{array}{l}\text { Candida } \\
\text { albicans }\end{array}$ & 15 & $\begin{array}{c}8.15 \\
\pm 1 . \\
3\end{array}$ & $\begin{array}{c}7.8 \\
\pm 0 . \\
6\end{array}$ & $\begin{array}{c}8.25 \pm \\
1.9\end{array}$ & $\begin{array}{c}7.75 \pm \\
2.7\end{array}$ & $\begin{array}{c}7.25 \pm \\
1.3\end{array}$ & $\begin{array}{c}7.3 \pm 1 \\
.2\end{array}$ & $\begin{array}{c}8.3 \pm 0 \\
.4\end{array}$ & $\begin{array}{c}9.1 \pm \\
1.0\end{array}$ \\
\hline $\begin{array}{l}\text { Aspergillu } \\
\text { s niger }\end{array}$ & 16 & $\begin{array}{c}6.5 \\
\pm 0 . \\
8\end{array}$ & $\begin{array}{l}6.9 \\
3 \pm \\
1.3\end{array}$ & $\begin{array}{c}7.0 \pm 0 \\
.3\end{array}$ & $\begin{array}{c}6.1 \pm 0 \\
.0\end{array}$ & $\begin{array}{c}6.1 \pm 0 \\
.0\end{array}$ & $\begin{array}{c}6.1 \pm 0 \\
.0\end{array}$ & $\begin{array}{c}7.35 \pm \\
2.1\end{array}$ & $\begin{array}{c}7.7 \pm \\
1.8\end{array}$ \\
\hline
\end{tabular}

Values are presented as the Mean \pm SE $(n=3)$

Due to the exceptional properties, probiotic bacteria may also have competed for nutrients (Marteau et al., 1990), and at the same time produced hydrogen peroxide and bacteriocins that performed as antibiotic agents (Wolfson, 1999). Excluding, bacteriocins, numerous probiotics also competent of reuterine production that is well-known to act as an antibacterial compound (Ray, 1996).

Finally, the capacity of the probiotics incorporated in bioyoghurts to inhibit the growth, or even kill certain selected pathogens confirms the health benefits one derives from the consumption of these yoghurts. Taking of these products can help protect one from occurrences of diarrhea, food poisoning and even systemic and enteric infections. The verified antibacterial effects of the probiotics supports the development of these functional foods as a key to the improvement of the health in the consuming public.

\section{Acknowledgment}

The authors are grateful to the Department of Pharmacy, East West University for valuable support during the progression of laboratory work.

\section{References}

Barefoot, S. F., \& Klaenhammer, T. R. (1984). Purification and characterization of the Lactobacillus acidophilus bacteriocinlactacin B. Antimicrob. Agents Chemother., 26, 328-334.

Heller, K. (2001). Probiotic bacteria in fermented foods: Product characteristics and starter organisms. Am. J. Clin. Nutr, 73, 374S-79S. 


\section{IIUC STUDIES, 14 (Issue 1, Special)}

Hill, C., Guarner, F., Reid, G., Gibson, G. R., Merenstein, D. J., Pot, B. L., Morelli, R. B., Canani, H. J., ... Sanders. (2014). Expert consensus document. The international scientific association for probiotics and prebiotics consensus statement on the scope and appropriate use of the term probiotic. Nature reviews. Gastroenterology \& hepatology, 11(8), 506-14.

Isolauri, E., Juntunen, M., Rautanen, T., Sillanaukee, P., \& Koivula, T. (1991). A human Lactobacillus strain (Lactobacillus casei sp. Strain GG) promotes recovery from acute diarrhea in children. Pediatrics, 88, 90-97.

Klaenhammer, T. R., \& Kullen, M. J. (1999). Selection and design probiotic. Int. J. Food Microbiol, 50, 45-57.

Magdalena, A. (2006). Probiotics in food: Health and nutritional properties and guidelines for evaluation. FAO Food and Nutrition paper 85, pp. 1-50.

Marteau, P., Pochart, P., Flourie, B., Pellier, P., Santos, L., Desjeux, J. F., \& Rambaud, J. C. (1990). Effect of chronic ingestion of a fermented dairy product containing Lactobacillus acidophilus and Bifidobacteriumbifidumon metabolic activities of the colonic flora. Am. J. Clin. Nutr., 52, 685-688.

Ouwehand, A. C., \& Vesterlund, S. (2004). Antimicrobial components from lactic acid bacteria. Food science and technology-New York-marcel dekker, 139, 375-396.

Ray, B. (1996). Health benefits of beneficial bacteria. Fundamental Food Microbiology (pp. 107, 194-97). USA: CRC Press.

Rijkers, G. T. (2011). Health benefits and health claims of probiotics: Bridging science and marketing. British Journal of Nutrition, 106(9), 1291-1296.

Wolfson, D. (1999). A probiotics primer. Nutrition Science News.

\section{Corresponding author}

Mohammed Abu Sayeed can be contacted at: pmasayeed@yahoo.com 